\title{
Potential Use of Cellular Stromal Vascular Fraction in Post-COVID-19 Pulmonary Injury and Adult Respiratory Distress Syndrome
}

\author{
Robert W. Alexander ${ }^{\star}, \dagger$
}

United States of America; University of Washington (Seattle), Department of Surgery, School of Medicine 83 Dentistry Global Alliance of Regenerative Medicine

DDOI: https://doi.org/10.15520/jcmro.v3i05.296

Accepted 25-05-2020; Received 10-05-2020; Publish Online 00-05-2020

\author{
Reviewed By: \\ Dr. Marwa M. Abu \\ Ali Department: \\ Reviewer/CMRO
}

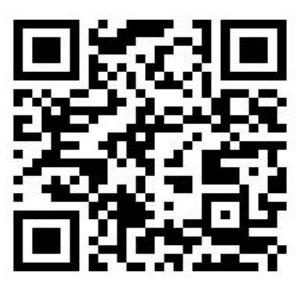

\begin{abstract}
A coronavirus (nCoV-2) has caused a worldwide pandemic, beginning in Wuhan, China. Patient's acquiring viral infection (COVID-19) which is often featured by elevation of temperature $\left(>100.3 \mathrm{~F}^{\circ}\right)$, cough, severe fatigue, and a variety of symptoms mimicking the flu and pneumonias. This gradually may extend to a progressive and serious respiratory failure with associated ARDS-like status and severe pneumonias with residual scarring of the interstitial areas of the lung alveoli, resulting in acute life-threatening, and permanent compromised gas exchange. Those that survive with controlled ventilation (low tidal volumes), medical induced coma, and prone positioning, are often left with reduction in pulmonary function abilities. This paper reviews some pertinent knowledge and advances in understanding of the pulmonary injuries and long-term damage of the COVID-19 patients, and reports a study to ameliorate such damage with use of cSVF for those who successfully recover.

There now exists technology to perform sophisticated analytics (Functional Respiratory Imaging, FRI) developed by Fluidda (Belgium, EU) which provides great detail and information, including the ability to help triage the infected patients early to help assist in prediction of ventilation and ICU needs. In addition, author's experience with use in Chronic Obstructive Pulmonary Disease (COPD) and Fibrotic Lung Diseases, the ability to evaluate effectiveness of use of cellular Stromal Vascular Fraction (cSVF) following intravascular deployment of isolated and concentrated cSVF. The hypothesis is that reduction of the widespread pulmonary inflammatory and immune responses which destroy much of the gas exchange abilities in the acute and residual damage which appears to create a long-term problem in the surviving patient population. Fluidda imaging analytics, coupled with standard pulmonary function studies, are effective at predictive studies which may prove of great help in anticipating which patient group is at most risk in the earlier infections to require ICU and potential ventilation needs.

Use of Stromal Vascular Fraction Cells (cSVF) are known to help mitigate damage from severe inflammatory disorders, provide immunomodulation, and promote repair and regenerative effects. The ease of access, simple ability to obtain significant numbers of viable cell elements, and the deployment via intravascular route has proven safe and efficacious in other forms of lung disease, including fibrotic lung disorders.

This paper reviews many of the current issues facing the severely infected PostCOVID-19 patient's lung compromise, and a therapeutic option which clearly shows promise to reverse some residual damage of the lung and gas exchange functions.
\end{abstract}

\section{INTRODUCTION:}

* Corresponding author.

$\dagger$ Email: rwamd@cybernet1.com
COVID-19 is a novel Coronavirus strain (SARS-CoV-2) that was first discovered in Wuhan, China in 2019. The 
virus has displayed properties of having a high transmission (human-to-human) rate via contact with infected individuals, particularly through coughing/sneezing and the nasopharyngeal mucosa. Studies have shown that the virus can remain viable in aerosols for many hours, and on various surfaces for many days. Furthermore, the virus has demonstrated that it impacts individuals at various degrees, often related to age and co-morbidities. For example, infected individuals may be totally asymptomatic, produce flu-like symptoms, or advance to full acute respiratory distress syndrome (ARDS) where they require non-invasive to full mechanical ventilation. These factors have contributed to the worldwide spread of infections, and by March 2020, the world health organization (WHO) has declared the virus a pandemic. Figure 1

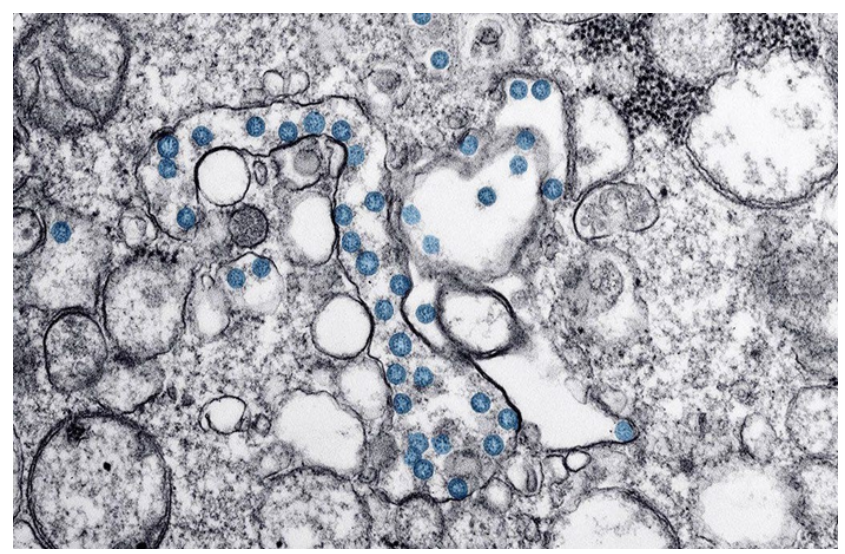

Figure 1. EM SARS-CoV-2 Virus

According to the data obtained from countries such as China, South Korea and Italy (some of the first epicenters of the pandemic), there is a general consensus that COVID-19 most severely impacts individuals that have an underlining condition (e.g. cardiovascular and respiratory diseases). immunodeficiency disorders, diabetes, and individuals above 50 years of age. Although this remains a valid opinion, cases of a few children and younger adults have encountered the disease, resulting in respiratory failure and cause of deaths. Immune system failure in the most severe cases seem to be more inclined to develop ARDS. [1] In situations where lung reserve capacity (used up the redundancy of the lungs to a point where they experience a critical, precipitous decline in function) result in emergency requirement of at least supplemental oxygen (O2), sometimes leading to the point of mandatory mechanical ventilation (up to EMCO use) in order to maintain acceptable $\mathrm{O} 2$ saturation. In later stages, the immense inflammatory reaction (following cytokine storm reaction to the virus) and immune response interferes with the gas exchange functions leading to somewhat unique form of life-threatening Acute Respiratory Distress Syndrome often resulting in loss of life. [2, 3]

Initially, most critical care and intensivists approached the severe COVID-19 patients as a serious pulmonary viral infection with challenging symptoms of ARDS. It became apparent, that the viral impact demonstrated classic ARDSlike pulmonary symptoms, which progressed in a somewhat different pattern requiring modification of care. At the time of this writing, it appears that the SARS-CoV-2 virus does enter via the lung system, where its changes are now considered classic in appearance on CT scans, particularly in the moderate to severe patient presentation. It is beginning to appear to have a progression somewhat dissimilar to classic ARDS, in spite of improved ICU management in very severe cases. These patients are noted to have a longer term ICU stay and enhanced ventilatory needs than expected. In addition to variable responses to care, there was discovered a significant disseminated intravascular coagulopathy (DIC), resulting in micro-thrombus formations with the small vasculature (distal extremities, kidney, liver, heart, brain, etc.). These appeared to compound the severity of disease, even in patients with few or no co-morbidities. Observations confirmed these patients experienced gradually increasing ddimer levels and a lowered platelet concentration, indicating a compounding issue of intravascular coagulation. [4]

When coupled with the serious Oxidative Stresses that were developing, compounding the tissue damage and systemic complications (including loss of limb, myocardial infarction, stroke, etc.). When treated with unfractionated heparin (UFH), the patients tended to respond favorably. This has been suggested as a result of the intravascular coagulopathy, with it's often highly elevated d-dimer levels, elevated circulating von Willebrand's Factor (vWF), and interaction with circulating Factor 8 were shown. This is consistent with the fact that this Cononavirus targets ACE2 receptors found in both the lungs and endothelial cells of vessel walls. With endothelial damage was incurred, escape of resident subendothelial vWF encountered Factor 8 and likely contributed to the blood coagulopathy. Some have suggested that this is consistent with the fact that the spike proteins of the Coronavirus targeted the ACE2 receptors located in the thin lining endothelial cells of not only vascular walls containing such receptors, but also present within the lung alveolar Type II cells. This explain how it not only impacts the lung, but also involves the delicate endothelial capillaries in the area of gas exchange. In disease progression, the finding of a DIC then accentuates the damage to the important interstitial lung alveolar areas, resulting in lowering of 02 saturation levels in the face of adequate ventilation efforts. This may reflect a reason for prolonged ICU and ventilation modification requirements in the severe COVID-19 patients.

As early as 2002, Loscalzo presented a paper explaining the interaction of Oxidative Stress to endothelial cell dysfunction and intravascular thrombosis. In this contribution, he presented a concise explanation of the damage resulting from excess reactive oxygen species (ROS) accumulations, and impacted the resultant atherothrombotic and vascular risk increases. [5] As more knowledge is found in the study of the COVID-19 pandemic, it is becoming apparent that the viral impact extends well beyond the alveolar lung and gas exchange, but may be further complicated systemically due to intravascular damage and resultant coagulopathy. 
This damage represents a reasonable target for the use of cSVF in this project to mitigate and potentially help repair the damaged gas exchange areas of the involved lung tissues, based on positive clinical experiences in COPD and IFL disease patients which are continuing to be studied in clinical trial at this time.

The pathogenesis of ARDS (as instigated by COVID-19), begins with the virus entering the human body and infecting the respiratory tract, more specifically attacking the lower airways which are believed to have a higher concentration of ACE-2 receptors (entry point for the corona-virus). Viral replication and subsequent cytokine storm release, triggers a major widespread inflammatory and immune reaction within the gas exchange region of the lung, eventually filling the alveoli with a proteinaceous material and loss of surfactant which effectively interrupts the capillary/alveolar portion of the lung leading to loss of $\mathrm{O} 2$ saturation and exchange. The edema caused within the interstitial portions of the distal airway and alveoli becomes massive, and is easily represented in HRCT lung or chest studies. Use of Fluidda FRI analyses has led us to a better understanding of the important differences in the ARDS classically encountered and the ARDS associated with COVID-19 patients. It is believed that COVID-19 group often initially report no major difficulty in their effort to breathe relatively easily, however, with lowering $\mathrm{O} 2$ saturations, some degree of confusion and signs of low oxygenation are encountered. It appears that a significant feature in COVID-19 related ARDS involves vascular dilation and pulmonary edema often mimicking findings of high altitude pulmonary edema patients (HAPE), often showing interstitial and alveolar fluid accumulation with accompanying mental confusion. It may explain why patients requiring hospitalization may be somewhat improved on $\mathrm{O} 2$ supplement via non-invasive modalities (including need for high flow or BIPAP use) at time of presentation to the Hospital. In summary, these may progress to more serious impairment of one, or all, gas diffusion elements caused by vascular inequality of Ventilation/Perfusion (V/Q), with development of pulmonary shunting, and interstitial barrier changes lowering effective gas exchange. Wagner (2008) suggested the use of Multiple Inert Gas Elimination Techniques (MIGET) to quantify V/Q inequality and pulmonary shunting, including the ability to evaluate extra-pulmonary influences (including cardiac output, body temperature, etc.). [6]

Some advance to the requirement of mechanical ventilation which must be managed somewhat differently than ARDS in severe pneumonias where compliance is often compromised and the patient's progress to struggling to ventilate. Approximately one-third of moderate-severe COVID19 patients have relatively normal or slightly lower compliance, but dropping $\mathrm{O} 2$ saturations in spite of supplementation. [7] These patients are vulnerable to ventilator-related pulmonary damage, where use of standard critical care protocols may be harmful and lead to complications. Overuse of high tidal volumes, higher PEEP pressures, and often improper positioning (supine versus using more prone positioning) may actually be over-distending the lungs. This results in worsening the ARDS condition, resulting in a fluid leak into the alveoli. This contributes to further gas exchange area damage (with infiltration of fibroblasts and scarring) or rupture of alveoli, producing irrevocable damage to the lung and for those who survive, a long-term lung dysfunction. In the 2013 New England Journal of Medicine, Dr. Guerin published the PROSEVA Study, a prospective, randomized controlled study reported the value of prone positioning in non-COVID-19 ARDS patients on standard ventilation protocols. These patients often did better and shortened the ventilatory demand faster than those place in a standard supine positioning. [8]

Early Chinese reports declared that CT was as accurate as early testing options in determining the diagnosis and, often, the prognosis. With the major advances and additional benefit from use of Fluidda analytics can now help to prognosticate the course of patients, and help analyze the safe and effective use of careful ventilation protocols which help tract required strict volume controls and carefully coordinated PEEP in order to prevent alveolar collapse and failure. Apparently in some individuals the highly reactive inflammatory-immune response may become rapidly overwhelming. These individuals exhibit severe inflammatory patterns within the alveoli, along with severe damage to the alveolar-capillary and interstitial gas exchange membrane and resulting in severe pulmonary edema. Figure 2

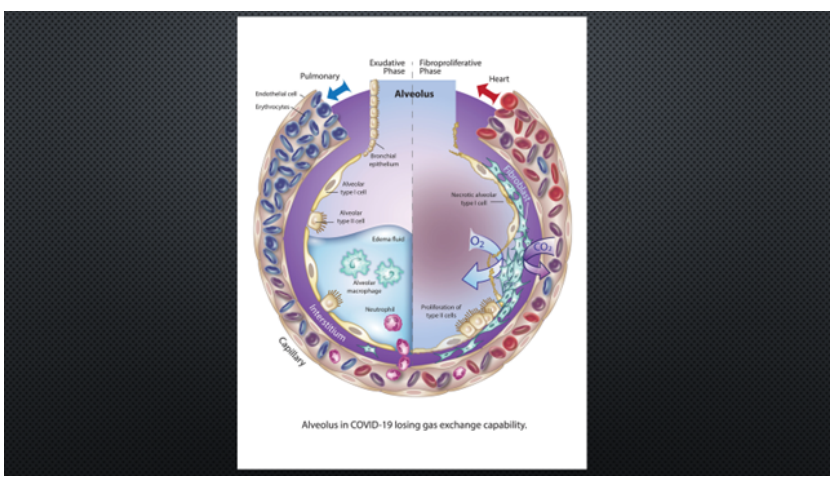

Figure 2. Diagrammatic Alveolus Phases in COVID-19 ChangesIn Gas Exchange Capability: Left Side =Exudative Phase Showing PA dilation, infiltration of fluid due to inflammation\& immune reaction (cytokine storm) into alveolus, including WBC,Macrophages, NK, etc. of innate immune Group; Right Side $=$ Fibroproliferation Phase showing poor gas exchange, damageto Alveolar Type I epithelial cells, Proliferation Alveolar Type II cells(surfactant), Fibroblastic infiltration \& thickening of Interstitial Spaceimpeding effective gas exchange. (C) [Illustration by Steven Tatman, San Diego, CA,USA]

Frequently, the best course of treatment for these individuals is a modified protocol for positive end expiratory pressure (PEEP) from non-invasive or mechanical ventilation in order to even maintain oxygenation levels needed. [9] Often, this requires an induced coma in order for the patients to not try to "override the ventilator", and achieve the best possible ventilation/perfusion capabilities. When patients are required to go on mechanical ventilation, it is generally considered that use of controlled low tidal volumes, induced coma, and use of mostly prone positioning are the optimal 
conditions needed to salvage the patients. There is clinically significant evidence that moderate to severe patients (hospitalization/ICU) that have recovered from their COVID19 infection; but still have mild to severe lung impairment due to the extensive response to the viral attack, including fibrotic changes that are permanent compliance and gas exchange issues.

Therefore, we propose a study utilizing strictly autologous adult cellular stromal vascular fraction (cSVF) as a potential interventional method for this population group demonstrating lung dysfunction residua. The use of autologous, stem/stromal cell elements in the cSVF are known to contribute to mediation of the inflammatory and immune reactions within the body via cell types, cellular secretions, and signaling (commonly known as "paracrine" functioning) to damaged tissues, and therefore, are potentially ideally suited to help mitigate this situation. [10] The heterogeneous cellular population of cSVF consists of cells important to the innate immune system (neutrophils, macrophages, monocytes, dendritic cells, NK cells, mast cells , etc.), which are the first reactors to any foreign antigen encounter, and serve to "present" the foreign elements to the adaptive immune system. The adaptive immune system then often can produce protective antibodies, effectively eliminates pathogens by preventing replication and often provide long-term protection in case of future exposures, often by creation of specific antibodies. [11] Elements of the adaptive immune system (T-regulatory, B-lymphocytes (marrow origin), and varieties of T-Cells (lymphoid tissues) are thought to be the key to the future resistance to re-exposure to specific antigens. Some of these cells are found within the cSVF, but more are attracted (particularly T-regulatory group) or created in, or near, the sites of infection or foreign proteins by periadventitial (pericytes, endothelial, adventitial) and mesenchymal cells. By introduction of these cSVF components, it is generally recognized that many "home" to the residual damaged and inflamed areas to help mitigate the terminal scarring and restriction of function in the individual patient. [12] The basis for the value of cSVF comes from previous studies clearly showing the safety of such autologous cell and efficacy in dealing with scarified and chronic inflammatory disorders. Figure 3

It is suggested that there is value in incorporation of the use of the Fluidda functional respiratory imaging (FRI) analyses using HRCT Lung (inhalation - exhalation series) to evaluate the structural changes in pulmonary dysfunction. Although if lung $\mathrm{CT}$ is not feasible in the severely compromised patients, use of thin section CT Chest can be used for much of the information gathering. This information is recommended to be compared from baseline to six (6) month sampling to determine anatomical findings of the treatment efficacy (cSVF) in its mitigation of inflammatory damage and structural response in the residual damaged tissues with comparative pulmonary function studies. Figure 4

As adipose tissue complex (ATC) is a plentiful, safe, and minimally invasive resource for acquisition of the autologous multipotent Stem/Stromal Cells which are collectively

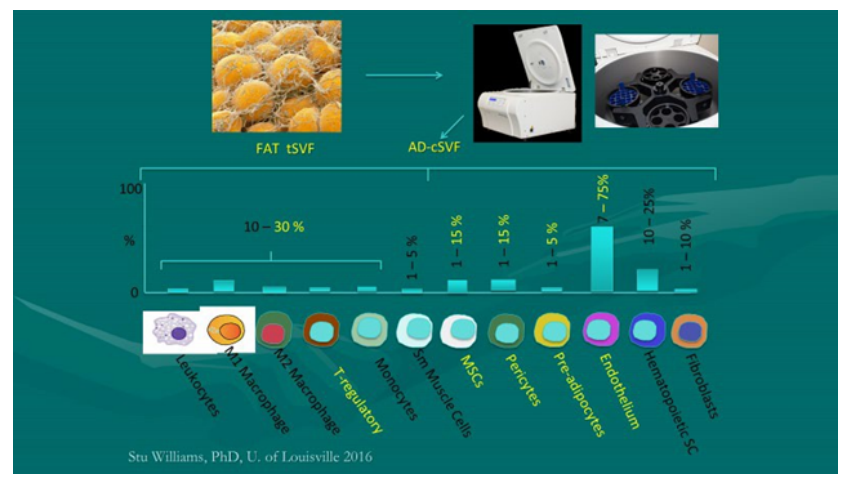

Figure 3. Upper Left: Image of Adipose Tissue Complex (ATC), Adipocytes (yellow) not in SVF, (fine mesh of perivascular periadventitial stem/stromal cells comprisingcSVF); Upper Right: Centricyte 1000, closed system for cSVFdigestive isolation, incubation/shaker table for concentration cSVF; Diagram ofHeterogeneous cell population of Stem/Stromal Cells of cSVF (Note: Comprised of cells of both innate andadaptive immunity

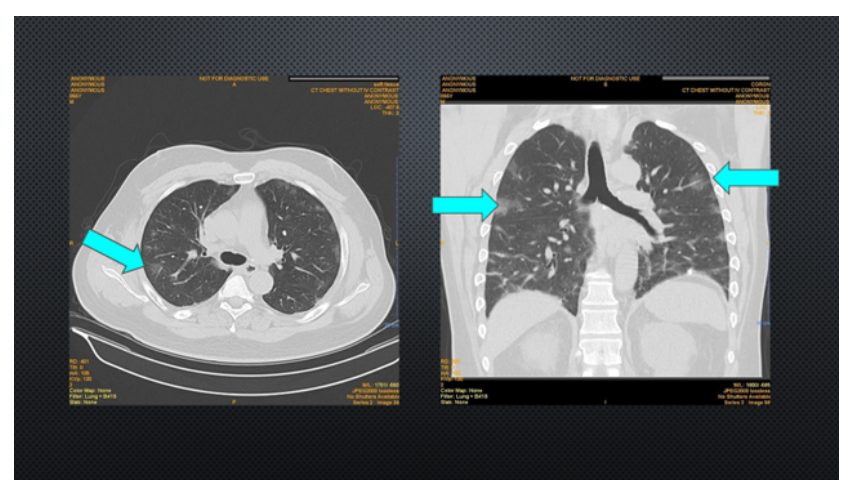

Figure 4. Moderate COVID-19 CT Chest. Ground Glass Appearance Of Peripheral Lesions (Blue Arrow Examples). [Images Provided by Michael Nissenbaum, MD,Interventional Radiologist]

referred to as Cellular Stromal Vascular Fraction (cSVF). It holds the largest depot of such heterogeneous cells in the body, and shown to have a combination of supportive cells and undesignated cells (autologous adult stem cells). They now have a demonstrated value in repair and regenerative capabilities important for the body to repair,heal and maintain its homeostasis activities. The concentration of these important reparative cells with ATC far exceeds that found from bone marrow origin (non-hematopoietic stem cell group). Figure 5

\section{MATERIALS AND METHODS:}

With the advent of sterile, coated, disposable, closed syringe microcannula system (GEMS, Tulip Medical Inc,, San Diego, CA, USA), gentle, safe and easy access to these cells and attachment matrix (ECM) has proven to be amongst the most reliable and trusted systems available. [13] The tiny cannulas (averaging $2.11 \mathrm{~mm}$ OD) allow the gentle, safe and effective means of harvesting the needed tissue to 


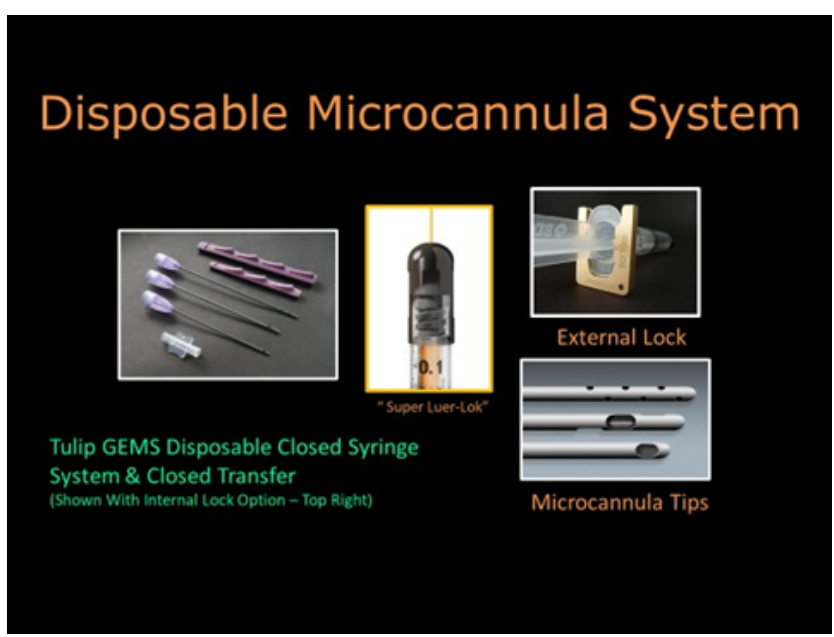

Figure 5. Disposable, Closed Syringe System UsingCoated Microcannula System (GEMS, Tulip Medical, San Diego, CA, USA. Low pressure acquisition of tSVF to permitisolation/concentration of cSVF. Bottom Right: Include $2.1 \mathrm{~mm}$ OD infiltration (multipleopenings) and harvesting (middle cannula).

provide millions of viable Stem/Stromal cellular elements. Usual volumes (30-35 cc including carrier fluid/dilute local solution) are then centrifuged (1000 g-force, $5 \mathrm{~min}$ ) to yield 20-25 cc of "compressed lipoaspirant". Digestive protocols for isolation of these cells from the adipose and matrix yielding a large number of freshly harvested and isolated/concentrated Stem/Stromal Cells for use. These are carefully evaluated for cellular numbers and viability (with RBC removal), and characterization via flow cytometry (ORFLO GO II, Ketchum, ID, USA). The cells are to be re-suspended in 500c sterile Normal Saline (NS), through in-line 150 micron filtration, via the intravenous route over 30 minute timeframe. In order to isolate and concentrate the cSVF, compressed ATC is enzymatically digested/incubated using a sterile collagenase blend (Type 1, Type 1l, and neutral proteases designed for ATC using Liberase TM (Roche Medical, USA) in a closed system processing system known as the Centricyte 1000 (Healeon Medical, Newbury Park, CA, USA) in accord with manufacturer's Instructions for Use. The processing and following centrifugation, effectively reduces the excess carrier fluids, is continued with addition of digestive enzymes utilizing an internal incubator/agitation cycle to insure efficient release of the stem/stromal cells, leaving the adipocytes/matrix. Following a proprietary neutralization and rinsing process (effectively removing all measurable residual enzymes), the specimen is re-centrifuged in the Centricyte 1000, pelletizing the cSVF via two stage centrifugation.

The autologous cellular pellet should be maintained at 37 degrees Centigrade until re-suspended in sterile NS for slow deployment via standard IV protocol as described above, with appropriate monitoring of patient's temperature, vital signs, and oxygen saturation. After deployment, the next major capillary bed that is encountered is that of the lungs, where ongoing Clinical Trials in COPD and Fibrotic Lung Disease, has shown evidence of safety and improvement of pulmonary functions and lowered requirements of supplemental oxygen. Not only is the analysis of detailed pulmonary function of value to the tracking progress of the lungs in COPD and Idiopathic Fibrotic Lung Disease, it is believed that examination of the damage and loss of pulmonary gas exchange capabilities may be reduced with use of cSVF. These changes, coupled with the permanent residual damage to the vascular-interstitial junction as a result of the immune and significant cytokine storm reaction from the viral insult may be amenable to use of cSVF in both the immunomodulation and mitigation of inflammation known features of the cSVF.

The cSVF components have been shown to compliment the process via cellular actions, but primarily via regional paracrine means. As the initial capillary bed is reached, it has been confirmed that some do engraft while others distribute systemically. The capabilities demonstrated in vitro, and in vivo situations trials, suggest there is a very good safety profile, and value in use of the autologous heterogeneous populations that comprise the SVF.

A recent pre-approval publication from the United Kingdom has recently observed that the autologous MSC populations examined in COVID-19 positive patients (from very severe, moderate and mild cases), which reported that the virus had not infected the undesignated group of MSCs. They report that this population is likely not susceptible to the virus as these undesignated cells do not display the ACE2 receptors on the cells. This suggests that the plentiful populations of MSC found in the SVF could still be very effective in therapeutic approaches such as is to be explored within this study. [14] A comprehensive FRI examination at baseline and 6 months is recommended to evaluate the ability of the cellular and paracrine signaling properties of the cSVF to favorably impact the pulmonary function abilities of post-viral infection patients currently enrolled in clinical trial (COVID-19).

Functional Respiratory Imaging (FRI) technology is considered a clinically applicable, non-invasive measurement for detailed analysis of patient specific respiratory systems. Using a set of distinct biomarkers permits analysis, exposure, structure, and function of the lungs and airways in a variety of respiratory diseases. This includes both obstructive and fibrotic lung disorders, with the opportunity to provide valuable and unique analytics which are of great value in establishing accurate functional and structural baselines for diagnosis coupled with progressive changes in clinical conditions with pharmacological or cellular therapies.

Use of the analytics, taken early in the diagnosis of COVID-19 may prove of great value in determination of early vascular changes (including arterial side pulmonary changes) which may be useful in predictive, prospective treatment of patients in the earlier stages of pulmonary decompensation. These have been instrumental in permitting customized, early non-invasive ventilation to adjusting full mechanical ventilation techniques most effective in COVID19 related ARDS. This facilitates modifications for each patient and their co-morbidities during the ICU requirement. The process begins with taking a low dose, high-resolution 
computerized tomography (HRCT) of the LUNGS (functional study). This functional study is taken in full inspiration and full expiration positions with rapid, thin-slice CT with attempted minimal radiation exposure. In addition, thin slice CT Thorax studies can permit analysis in those not capable of completing the lung study in inhalation and exhalation efforts. From those imaging sets, measurements are taken to permit a segmented, 3-dimensional geometries which accompany the computational fluid dynamics (CFD) to quantify airflow and distributions of exposure. [15-19]

The early evaluations of changes in the COVID-19 patients on average demonstrate they have significantly smaller diameters of small vessel $\left(\mathrm{CSA}<5 \mathrm{~mm}^{2}\right)$ compared to healthy and non-COVID-19 ARDS. This is of interest, as it suggests an unexpected vasoconstriction compared to the vasodilation noted the the non-COVID-9 ARDS patients. This is core evidence as to why careful use of tidal volumes and PEEP controls to avoid Ventilator-Induced Lung Injury (VILA) in these patients is important. This raises the question that use of inhaled Nitrous Oxide (iNO) may be of some value to alleviate the vasoconstrictive tendencies in COVID-19 ARDS earlier in cases may avoid the precipitous fall into hypoxia and require intubation.

\section{DISCUSSION:}

As the ongoing pandemic is in the process of mitigating the widespread infection, we must look forward to using recently developing technologies which may provide great value in dealing with present damage and reducing other related issues in the future. The ability to utilize the FRI testing, including use of inert gas elimination evaluation, both in prediction of course and in reducing the residual lung damage resulting from the ongoing viral challenges now and in the future. In addition to value in COVID-19, the same analytics can provide invaluable information in pulmonary conditions which impacts about 65 million people, representing the third leading cause of death in the world. These groups found of great value to the FRI analytics fall into both the obstructive (difficulty in exhalation) and fibrotic lung disorders (difficulty with compliance and inhalation).

As a major advance in the diagnosis, prognostication/tracking of both pharmaceutical and cellular therapies, and improvements in quality of lives, offers the ability to provide enhanced and customized management of many of these patients. These advances provide much more information and value than use of standard pulmonary function testing (PFT) to providers who encounter the obstructive and fibrotic lung disorders. Findings can be carefully analyzed and reported in order to determine the safety and efficacy of use of autologous stem/stromal cell elements and pharmaceuticals in forms of fibrous lung dysfunction as a result of the infection from SARS-CoV-2 virus. A controlled, randomized phase I-II study should be carried out following testing of this protocol effort proves successful as it has in COPD and Idiopathic Pulmonary Fibrosis.

\section{Acknowledgements:}

Special appreciation to Black Tie Medical for their Sponsorship in patient funding of this pilot project for the patients in need. Thanks to Pat Alexander, Susan Riley, and Nancy Smith for their support and role in patient management, tracking and provision of care.

Conflicts of Interest: No conflicts of interest of author noted

\section{REFERENCES}

1. Wu, K. Peng, G. et al. "Mechanisms of Host Receptor Adaptation By Severe Acute Respiratory Syndrome Due To Coronavirus", J. Biol Chem, (2012), 287 (12): 8904-8411.

2. Li, G., Fan, Y. et al, "Coronavirus Infections and Immune Responses”, J. Medical Virology, (2020), 92: 424-432.

3. Li, G., De Clercq, E. "Therapeutic Options for the 2019 Novel Coronavirus (2019-nCoV-2)”, Nat Rev Drug Discovery (2020), 19(3): 149-150, doi: 10.1038/d41573-020-00016-11.

4. Bi, X, Su, Z., et al., "Prediction of Severe Illness Due To COVID-19 Based On An Analysis of Initial Fibrinogen to Albumin Ratio and Platelet Count”, Platelets (2020), epub: 1-6.

5. Loscalzo, J., "Oxidative Stress in Endothelial Cell Dysfunction and Thrombosis", Pathophys, Hemostasis, Thrombosis (2002). Doi:https/doi.org/10-1080/09537104.2020.17600230.

6. Leng, Z, Zhu, R. et al. Transplantation of ACE2 Mesenchymal Stem Cells Improves the Outcome of Patients with COVID-19 Pneumonia. J. Aging \& Dis (2020), 11(2): 216-228.

7. Wagner PD. The multiple inert gas elimination technique (MIGET). Intensive Care Med. 2008;34(6):994-1001. doi:10.1007/ s00134-008-1108-6.

8. Gattinoni, L, Chiumello, D et al. COVID-19 Pneumonia: Different Respiratory Treatment for Different Phenotypes? J. Intens Care Med.(2020)

DOI:101007/s00134-020-06033-2.

9. Guerin, C., Reignier, J., PROSEVA Study Group, et al. "Prone positioning in Severe Acute Respiratory Distress Syndrome", N. Engl J Med (2013); 368: 2159-2168.

10. Schepens, T., Van Holsbeke, C. et al. Assessing Regional PEEPRelated Airway Opening and Dilatation in ARDS Patients Using Functional Respiratory Imaging (FRI). ERS (2015), 9: 1-19.

11. Alexander, Robert W., "Understanding Adipose-Derived Stromal Vascular Fraction (AD-SVF) Cell Biology and Use on the Basis of , Chemical, Structural and Paracrine Components, (2012), J. of Prolotherapy, 4: 855-869.

12. Atluri, S, et al. Expanded Umbilical Cord Mesenchymal Stem Cells as a Therapeutic Strategy In Managing Critically Ill COVID-19 Patients. Pain Physician (2020), 23: E71-83.

13. Alexander, Robert W., "Overview of Cellular Stromal Vascular Fraction (cSVF) \& Biocellular Use”. (2019), J Stem Cell Res Dev., S1003, DOI:28710.24966/SRDT-2060/S1003.

14. Alexander, R.W., Harrell, D.B., Autologous fat grafting: Use of Closed Syringe Microcannula System For Enhanced Autologous Structural Grafting, J. Clin, Cosmetic, and Investig Derm (2013), 6: 91-102.

15. Metcalfe, S. M. "Mesenchymal Stem Cells and Management of COVID-19 Pneumonia”, Med in Drug Discovery, (2020), https://doi.org/10/1016/j.medidd.2020.100019 
16. De Backer, J.W., De Backer, W. et al. Flow Analyses in the Lower Airways: Patient-Specific Model and Boundary Conditions. Med Eng \& Physics (2008), 30: 872-879.

17. Lanclus, M., De Backer, J et al. Machine Learning Algorithms Utilizing Functional Respiratory Imaging May Predict COPD Exacerbations. Acad Radiol (2018), 1-9, doi.org/10/1016.j.acra.2018.10.022.

18. Barbosa, E. Lanclus, M. et al. Machine Learning Algorithms Utilizating Quantitative CT Features May Predict Eventual Onset of Brochiolitis Obliterans Syndrome After Lung Transplantation. (2018), Acad Radiol, 25:1201-1212.

19. Clukers, J, Landus, M. et al. Quantitative CT Analysis Using Functional Imaging Is Superior In Describing Disease Progression In Idiopathic Pulmonary Fibrosis Compared to Forced Vital Capacity. (2018), J Respir Res 19: 213-222. Doi.org/10.1186/s12931-018-0918-5. 